\title{
Adherence to Disease-Modifying Therapies for Multiple Sclerosis
}

\author{
Lucas Higuera, MA; Caroline S. Carlin, PhD; and Sarah Anderson, PharmD
}

\section{ABSTRACT}

BACKGROUND: Multiple sclerosis (MS) is a neurological degenerative chronic condition without cure. However, long-term disease-modifying therapies (DMTs) help reduce the severity of MS symptoms. Adherence to DMTs is key to their success. Several studies have analyzed what makes patients adherent to their DMTs. As new DMTs have entered the market, few studies have analyzed factors of adherence using all currently available DMTs.

OBJECTIVE: To analyze different factors of adherence to DMTs for MS, in particular how the type of DMT affects adherence.

METHODS: This retrospective cohort study used enrollment and claims data from an upper Midwest health plan in the United States between 2011 and 2013. Patients entered the study if they had any medical claim with an MS diagnosis and used only 1 DMT during the study time frame. Medication possession ratios (MPRs) were computed as the fraction of days with medication supplied during the year; patients with MPRs of 0.8 or higher were considered adherent. Multivariate probit models with patient-specific random effects were estimated, with controls for demographic characteristics, type of DMT, health plan type, and measures of health status.

RESULTS: Patients aged over 45 years were between 13.7 to 18.6 percentage points more likely to be adherent than younger patients. Women had a 5.5 percentage-point lower probability of being adherent than men. Patients using self-injectable DMTs with injection site reactions as the most likely side effect were 9.1 percentage points less likely to be adherent than patients using oral, infusible, and other self-injectable DMTs. Patients with depression had a 5.5 percentage-point lower probability of being adherent. These results were robust to changes in controls for type of plan and neighborhood socioeconomic characteristics.

CONCLUSIONS: This study found statistically significant differences in adherence to DMTs by age, sex, type of DMT, and a depression diagnosis.

J Manag Care Spec Pharm. 2016;22(12):1394-401

Copyright $\odot 2016$, Academy of Managed Care Pharmacy. All rights reserved.

\section{What is already known about this subject}

Multiple sclerosis (MS) is a degenerative neurological condition that affects approximately 400,000 patients in the United States, with no cure at this time.

Disease-modifying therapies (DMTs) help reduce the severity of MS symptoms and slow disability progression.

Treatment adherence is key to the effectiveness of DMTs. Factors found to affect adherence to DMTs include cognitive functioning, convenience of use, side-effect profile, provider/caregiver support, and self-efficacy.

\section{What this study adds}

With data from a health plan that include privately and publicly insured patients, this retrospective study compared adherence to all available DMTs used to treat MS.

Not all self-injectable MS drugs are the same: the probability of being adherent to self-injectables with injection site reactions as the most likely side effect is 9.1 percentage points lower than selfinjectables with other side-effect profiles.

Patients with depression were less likely to be adherent.

$\mathrm{M}$ ultiple sclerosis (MS) is a chronic autoimmune disease affecting the central nervous system. The disease targets the myelin sheaths around nerves, leading to inflammation, myelin loss, and axonal destruction. MS is the most common cause of neurologic disability in people aged between 20 and 50 years. ${ }^{1}$ There are approximately 400,000 cases of MS in the United States and approximately 2.5 million worldwide. ${ }^{1,2}$ Unfortunately, the overall incidence of MS is increasing, with 200 individuals diagnosed in the United States each week. ${ }^{3}$

The course of MS can look different for every patient. The signs and symptoms experienced by each patient vary widely and may include visual problems, fatigue, paresthesia, bladder/ bowel/sexual dysfunction, gait problems, spasticity, dizziness, vertigo, pain, depression, and cognitive dysfunction. Some less common symptoms of MS include headache, hearing loss, itching, seizures, speech/swallowing difficulties, tremor, and loss of coordination. ${ }^{4}$ The medications used to treat the signs and symptoms of the disease do not produce a cure but can substantially improve symptoms, decrease disability progression, and improve quality of life.

There are 4 classifications of MS: relapsing-remitting multiple sclerosis (RRMS), primary progressive multiple sclerosis, secondary progressive multiple sclerosis (SPMS), and progressive relapsing multiple sclerosis. Disease-modifying therapies (DMTs) have been developed over the past 20 years and help to reduce the number and severity of relapses in patients with relapsing forms of MS but are ineffective in progressive forms of MS. However, these medications must be taken indefinitely and are not a cure for the disease, since patients can still experience relapses while using DMTs. The first injectable DMT came onto the market in 1993.5 The 13 current DMTs that are on the market include injectable immune modulators such as glatiramer acetate (Copaxone); interferon beta-la (Avonex, Refib); 
interferon beta-1b SQ and interferon beta-1b IM (Betaseron, Extavia); and the immune suppressants mitoxantrone (Novantrone) and natalizumab (Tysabri), which are administered as infusions. Since 2011, the first oral agents have been approved to treat RRMS—-fingolimod (Gilenya), teriflunomide (Aubagio), and dimethyl fumarate (Tecfidera). Additionally, peginterferon beta-la (Plegridy), generic Copaxone (Glatopa), and the infusible agent alemtuzumab (Lemtrada) have recently entered the market. The long-term safety profile of many of these newer oral agents is unknown because of the short time they have been on the market. These DMTs act to reduce the number and severity of relapses, reduce the disease activity seen with magnetic resonance imaging (MRI), and may reduce accumulation of disability in patients with RRMS ${ }^{6,7}$ Irreversible changes occur early in the course of MS; therefore, early detection and treatment is important. Because disease activity can occur "silently," periodic MRI monitoring should be considered, even in patients with minimal progression of observable symptoms.

An individualized approach to treatment and a shared decision-making process between providers and patients are necessary for patients to reach their own health goals. ${ }^{8,9}$ Some factors to be considered when making a treatment decision include comparative efficacy, safety, route of administration, side-effect profile, tolerability, dosing frequency, patient lifestyle factors, and payer formulary status. ${ }^{10}$ Literature on adherence to DMTs shows varying levels of nonadherence rates, depending on type of drug and mode of application..$^{11-13}$ Because of the complex and dynamic issues that the MS patient population faces, additional support from health care professionals is warranted.

The World Health Organization defines adherence as "the extent to which a person's behavior-taking medication, following a diet, and/or executing lifestyle changes_corresponds with agreed recommendations from a health care provider."14 Nonadherence includes "failing to fill prescriptions, delaying prescription fills, reducing the strength of the dose taken, and reducing the frequency of administration. It can also include the failure to keep appointments or to follow recommended lifestyle or dietary changes." ${ }^{14}$

Adherence to DMTs is a multifactorial problem within the MS population. According to a longitudinal, prospective study of 97 patients with definite MS who were taking a DMT, 73\% missed at least 1 dose; $10 \%$ missed more than 10 doses in a 6-month period; and 25\% stopped the DMT altogether. ${ }^{15}$ Typical dosing regimens for DMTs vary and can include daily, 3 times per week, weekly, and monthly. Patients with a history of missed doses are more likely to miss future doses. ${ }^{15}$ Nonadherence is shown to lead to more frequent relapses and decompensating health, which increase overall health care resource consumption. ${ }^{16,17}$ There are numerous and divergent factors influencing missed doses and discontinuation of DMT, ${ }^{15}$ which indicates the need for a multifaceted approach to improving adherence. There are many barriers contributing to adherence issues, including cognitive problems, ${ }^{18,19}$ fear of medication administration and inconvenience of use, ${ }^{12,16}$ lack of self-efficacy, ${ }^{12,20}$ misinformation and not understanding the disease ${ }^{19}$ inappropriate treatment perceptions or expectations, ${ }^{12}$ provider/caregiver support, ${ }^{21,22}$ side effects, ${ }^{13,16-18,22}$ insurance issues, ${ }^{23}$ increasing copays, ${ }^{23,24}$ and level of disability and comorbid conditions associated with MS. ${ }^{20,22,25-27}$ Comorbid conditions, such as depression, recurring urinary tract infections, and hypertension, are more prevalent among the MS population than the general public. ${ }^{28}$

Other studies compare adherence by type of DMT. There is no consensus on which DMT has higher adherence: some studies show that patients using interferon beta-la are more adherent ${ }^{16,29}$; others show that patients using fingolimod are more adherent ${ }^{30,31}$; and still others show a lower medication possession ratio (MPR) in patients using glatiramer acetate..$^{32}$ However, these studies do not compare the whole array of available DMTs. The purpose of this study was to compare adherence among all available DMTs for MS at the time of the study. The results of this study showcase several associations between individual characteristics of patients and adherence to the various types of DMTs.

\section{Methods}

\section{Data}

This retrospective study used 2011-2013 enrollment, medical claims, and pharmaceutical claims data from an upper Midwest health plan in the United States. Enrollment included commercial plans, individual and family health plans, managed Medicaid and Medicare plans, and dual Medicaid/Medicare (dual eligibility) plans. From the individuals enrolled in the health plans in 2011-2013, we selected MS patients for the study if they were aged 18 years or older, had any medical claim with an International Classification of Diseases, Ninth Revision, Clinical Modification code for MS (340 or 340.0), and had a prescription filled for an MS DMT in any year of data. Once patients were identified, information was collected on enrollment and medical and pharmaceutical claims for 2011-2013. From an initial population of 1,636,407 patients, 3,688 had an MS diagnosis code in their claims data, 1,231 of whom received any DMT. Measures of health risk and depression were lagged by 1 year to minimize statistical feedback between adherence and health status; 459 patients in the sample did not have any previous information; 48 patients had less than 6 months of enrollment on a given year; and 26 patients switched DMTs. The exclusion of patients who switched DMTs may have biased these results, but the small number of those patients suggested that this bias would be minimal. Figure 1 shows the sample selection process.

The informatics team merged in neighborhood characteristics from the 2011 5-year American Community Survey using patient address at the block group and census tract level. 


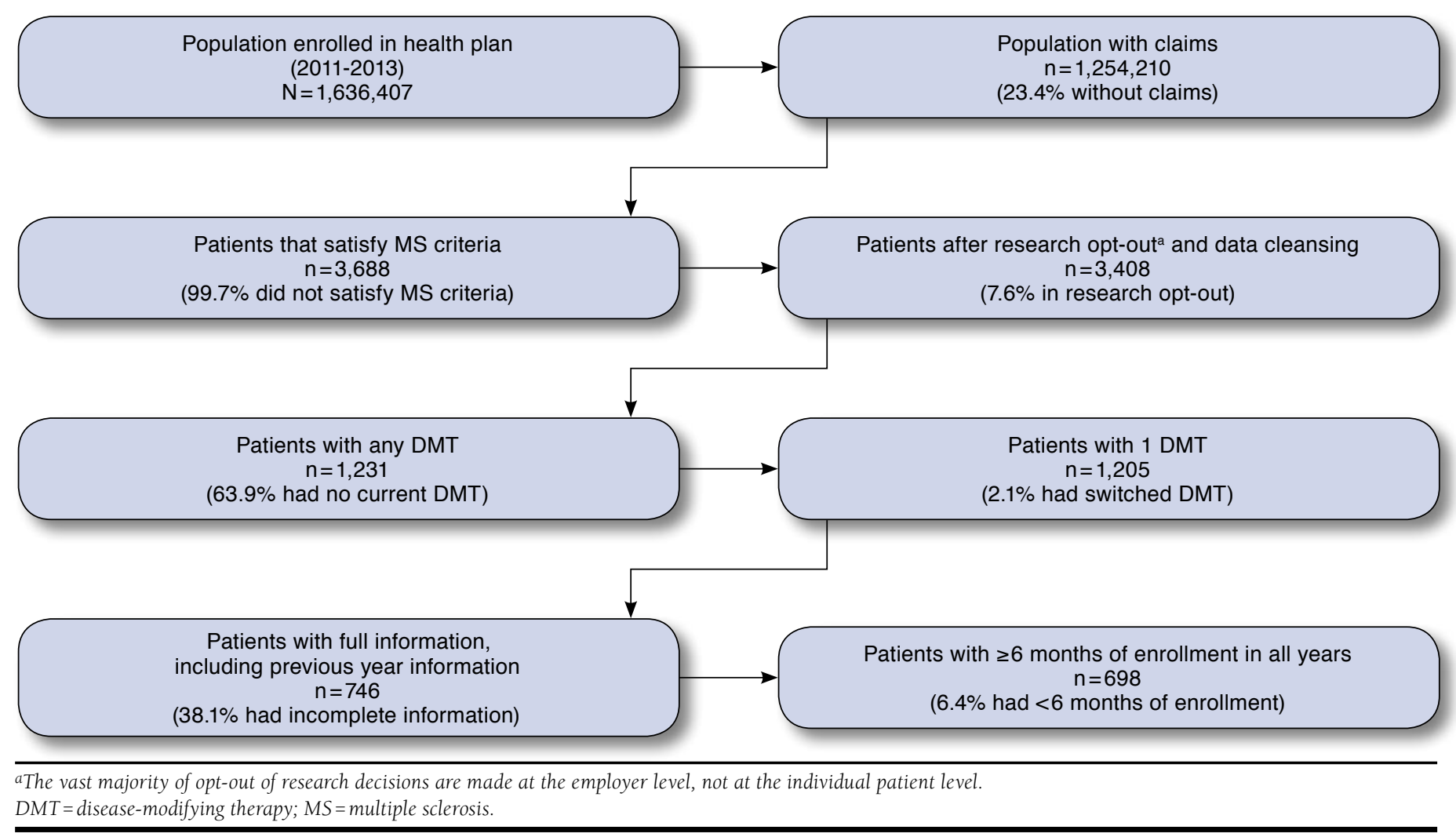

Neighborhood characteristics capture the socioeconomic environment in which patients functioned. The final sample contained patients aged 18 years or older, with a diagnosis of MS, who receive any DMT without switching therapies, who were enrolled in the health plan for at least 6 months, and who had health risk information for the year previous to the adherence measurement. The final, full-information sample consisted of 1,045 observations from 698 patients during 2012-2013. All data handling procedures followed the protocol submitted to the research team's external institutional review board, which determined that this study was exempt from review.

To assess each patient's health status, the resource utilization band was used in the Johns Hopkins Adjusted Clinical Groups (ACG) system. The ACG system categorizes patients into 6 levels of prior-year health risk ("no usage," "healthy user," "low risk," "medium risk," "high risk," or "very high risk") according to health care use during the year. Because the sample for this study was disproportionally biased towards high-risk patients, patients were categorized into either "no usage through medium risk," or "high risk/very high risk" in the previous year. Additionally, the ACG system identifies patients with chronic conditions, and following previous studies, ${ }^{33,34}$ prior-year depression was included as a factor contributing to adherence. We calculated the measures from the ACG system using health care use of 2011-2012 but included in the analysis measures lagged 1 year. By restricting the sample to years in which individuals had exposure of 6 months or more, we made sure the health use patterns in the ACG system measures had enough information to be reliable.

Patients with comorbidities or other chronic conditions may seek more care from primary care than from specialists, but specialists may be more fit to manage patients with MS. In order to analyze these possible effects of different styles of disease management or differences in management of comorbidities and other chronic conditions, a variable was included that indicated whether more of a patient's evaluation and management $(E \& M)$ visits were from a primary care physician than from a neurologist.

\section{Measures and Factors of Adherence}

We identified 8 different DMTs and categorized them into 4 groups according to their routes of administration and most common side effects. Table 1 summarizes the characteristics of these DMTs. The pharmacy claims included all claims for injectable and orals, under the corresponding National Drug 
TABLE 1 Disease-Modifying Treatments Included in Study ${ }^{a}$

\begin{tabular}{l|c|c|c|c}
\hline \multirow{2}{*}{$\begin{array}{l}\text { Category } \\
\text { Self-injectable, } \\
\text { flu-like side effects }\end{array}$} & Trade Name & Generic Name & \multicolumn{1}{|c|}{ National Drug Code Numbers } & \multicolumn{1}{c}{$\begin{array}{c}\text { Current Procedural } \\
\text { Terminology Codes }\end{array}$} \\
\cline { 2 - 5 } & Avonex & Interferon beta-la & $\begin{array}{l}59627-0002-05,59627-0002-07,59627-0003-04, \\
59627-0001-03,59627-0001-04\end{array}$ & J1826, Q3025, Q3026 \\
\cline { 2 - 5 } & Rebif & Interferon beta-la & $\begin{array}{l}44087-0022-03,44087-0044-03,44087-8822-01, \\
44087-3322-01,44087-3344-01,44087-0188-01\end{array}$ & J1826, J1830, Q3025, Q3026 \\
\cline { 2 - 5 } $\begin{array}{l}\text { Self-injectable, } \\
\text { skin side effects }\end{array}$ & Copaxone & Glatiramer acetate & $68546-0317-30$ & J1830 \\
\hline Orals & Gilenya & Fingolimod & $00078-0607-51$ & J1595 \\
\cline { 2 - 6 } & Aubagio & Teriflunomide & $58468-0210-02,58468-0211-01$ & Not available \\
\cline { 2 - 5 } & Tecfidera & Dimethyl fumarate & $64406-0005-01,64406-0007-03,64406-0006-02$ & Not available \\
\hline Infusions & Tysabri & Natalizumab & $59075-0730-15,64406-0008-01$ & J2323 \\
\hline
\end{tabular}

aThere were only 10 disease-modifying treatments on the market at the beginning of this study. Very few claims were identified for Extavia, and none were identified for Novantrone.

Code numbers. Medical claims included infusible DMTs and other injectable DMTs identified with J- and Q- Current Procedural Terminology codes, since they are usually administered in a clinic/facility setting.

Adherence was the outcome of interest, which was measured using individual MPRs for each year that patients were present in the data. Patients were observed every year that they were enrolled in the health plan for 6 months or more and satisfied the eligibility criteria previously described. We had a "prevalent user" design, in which 337 patients (48.3\% of the sample) had a prescription to any DMT at the beginning of the study time frame, and the remaining 361 initiated a DMT during the study time frame. This design biased upwards the adherence rates, since the sample was selected by not observing those patients who may have already stopped taking DMTs. ${ }^{35}$

The denominator of the MPR was the number of days in the year after the prescription was initiated, adjusted for loss of coverage if the patient did not have continuous health plan coverage through the end of the year. Since interferon DMTs were supplied in 28-days intervals, we adjusted the number of days in a year to $28 \times 12=336$ (alternative methods to adjust the number of days for interferon DMTs yield very similar MPRs and adherence rates). The numerator was the number of days supplied of the medication. The number of days supplied was determined by the amount of the medication dispensed in combination with the dosage frequency. If the days supplied on a fill date exceeded the number of days left in the calendar year, the excess days were carried over to the following year. Therefore, the MPRs could not be lower than zero or higher than 1 . Since it is standard in the literature, ${ }^{36}$ a patient was defined as adherent to a medication if the MPR was 0.8 or above.

\section{Statistical Analysis}

Multivariate probit models were estimated to identify factors associated with DMT adherence, in particular the difference in adherence among the different DMT types. Because the data included repeated observations of patients over time, we allowed for patient-specific random effects and clustered standard errors at the patient level. ${ }^{37}$ Clustered standard errors correct for the within-group correlation of repeated observations per patient. The dependent variable was a binary indicator of adherence (MPR $>0.8)$. The benchmark model included age, sex, DMT type, type of health plan, prior-year health risk, a measure of whether the majority of the E\&M visits were with a primary care physician or a neurologist, and the prior-year depression indicator as independent variables. To avoid any feedback effect of higher adherence into a lower health risk (because of better treatment efficacy ${ }^{17}$ ) or a higher chance of depression (as a side effect of the DMT), health risk and depression were lagged 1 year in the model (e.g., to explain the 2013 adherence measure of a patient, the model included health-risk and depression measures from 2012 and the remaining information measured in 2013).

Logistic regression models are often used to analyze binary outcomes, such as adherence. The estimated coefficients of a logistic regression show the direction and statistical significance of the correlation between the dependent variable and the independent variables but provide no intuitive interpretation of the magnitude of the correlation. Because of this, the estimated coefficients of a logistic regression are often transformed into odds ratios. However, odds ratios are difficult to understand and easily misinterpreted as a change in the likelihood of the outcome. ${ }^{38}$ Probit regression models are an alternative to logistic models, but estimated coefficients in probit models also do not provide intuition about the magnitude of the correlation. We used a probit model and also used the estimated coefficients to compute marginal effects, which are percentage-point changes in the estimated probability of the binary outcome associated with a change in each independent variable. Marginal effects are easier to understand, providing an intuitive measure of how adherence is associated with the independent variables. 
TABLE 2 Descriptive Statistics, 2012-2013

\begin{tabular}{|c|c|c|c|}
\hline & Observations & Mean & SD \\
\hline MPR & 1,045 & 0.7799 & 0.415 \\
\hline \multirow[t]{2}{*}{ Adherence (MPR $\geq 0.8$ ) } & 1,045 & 0.8718 & 0.224 \\
\hline & Observations & Percentage & $\begin{array}{c}\text { Number of } \\
\text { Patients }\end{array}$ \\
\hline \multicolumn{4}{|l|}{ Age category, years } \\
\hline $18-34$ & 1,045 & 12.2 & 127 \\
\hline $35-44$ & 1,045 & 20.4 & 213 \\
\hline $45-54$ & 1,045 & 29.1 & 304 \\
\hline $55-64$ & 1,045 & 29.4 & 307 \\
\hline $65+$ & 1,045 & 9.0 & 94 \\
\hline Female & 1,045 & 74.9 & 783 \\
\hline \multicolumn{4}{|l|}{ DMT type } \\
\hline Injectable, flu side effects & 1,045 & 42.7 & 446 \\
\hline Injectable, skin side effects & 1,045 & 44.5 & 465 \\
\hline Orals & 1,045 & 4.1 & 43 \\
\hline Infusions & 1,045 & 8.7 & 91 \\
\hline \multicolumn{4}{|l|}{ Health plan } \\
\hline Commercial & 1,045 & 73.4 & 767 \\
\hline Medicaid only & 1,045 & 8.9 & 93 \\
\hline Medicare/Medicaid & 1,045 & 1.1 & 12 \\
\hline Medicare only & 1,045 & 16.6 & 173 \\
\hline \multicolumn{4}{|l|}{ Health status, previous year } \\
\hline $\begin{array}{l}\text { No use/healthy/low/ } \\
\text { medium risk }\end{array}$ & 1,045 & 57.8 & 604 \\
\hline High/very high risk & 1,045 & 42.2 & 441 \\
\hline E\&M: primary care $>$ neurologist & 1,045 & 74.7 & 781 \\
\hline Depression, previous year & 1,045 & 52.6 & 550 \\
\hline $\begin{array}{l}\text { Neighborhood: \% of } \\
\text { non-Hispanic whites }\end{array}$ & 977 & 86.8 & 848 \\
\hline \multicolumn{4}{|l|}{ Neighborhood education levela } \\
\hline More than high school & 977 & 65.7 & 641 \\
\hline High school & 977 & 26.6 & 260 \\
\hline Less than high school & 977 & 7.7 & 76 \\
\hline Neighborhood: \% below FPLa & 977 & 9.4 & 92 \\
\hline Rural & 1,045 & 16.1 & 168 \\
\hline
\end{tabular}

${ }^{a}$ Geographical information was not complete for all patients.

$D M T=$ disease-modifying therapy; $E \& M=$ evaluation and management; $F P L=f e d-$ eral poverty level; $M P R=$ medication possession ratio; $S D=$ standard deviation

In addition to the benchmark model for this study, 3 additional models were estimated as robustness tests of the main results. Not all types of DMTs were available during the 3 years of data. The oral DMTs teriflunomide and dimethyl fumarate were approved by the U.S. Food and Drug Administration as DMTs indicated for MS in September 2012 and March 2013, respectively. ${ }^{39,40}$ Because of the recency of their approval, oral DMTs were present in our data in low frequencies, primarily in 2013, and their impact on adherence was imprecisely measured. Therefore, our first robustness check evaluated how the results change when patients taking oral DMTs were excluded from the comparison.
Socioeconomic characteristics may drive adherence results and choice of DMTs, confounding the difference in adherence among DMTs. For instance, patients with more difficult access to medical facilities could be more likely to choose oral DMTs over infusible products and simultaneously have lower adherence. Our second and third robustness checks excluded patients in Medicaid and Medicare/Medicaid managed plans and added neighborhood socioeconomic characteristics as independent variables.

\section{Results}

Table 2 shows the descriptive statistics of the MPRs, the adherence measure, and other patient and neighborhood characteristics used in the statistical analysis. Compared with Halpern et al. (2009), ${ }^{29}$ who followed a "new user" research design, the MPR and the percentage of adherent patients in this study were 0.05 points and 29.3 percentage points higher, respectively. However, Halpern et al. only included commercially insured patients and did not include oral and infusible DMTs.

Table 3 displays the estimated marginal effects, with their $P$ values and 95\% confidence intervals noted. The estimates show the change in the probability of being adherent associated with the independent variable. For example, the marginal effect equal to 0.077 in the age range of 35-44 years indicates that a patient in this age range had a 7.7 percentage-point higher estimated probability of adherence, compared with a similar patient in the reference age range of 18-34 years. Compared with patients aged 18-34 years, patients aged 45 years or older had a 13.7 (aged 45-54 years) to 18.6 (aged 65 years or older) percentage-point higher probability of being adherent. Women had an estimated probability of being adherent that was 5.5 percentage-points lower compared with men. When comparing DMT types, patients who took self-injectable DMTs and whose most common side effect was injection site reactions were 9.1 percentage points less likely to be adherent than those in the reference group (those who took self-injectable DMTs and whose most common side effect was flu-like symptoms). In robustness checks (available from the corresponding author), the DMTs with injection site reactions were separated into the individual DMTs, with consistent results. Patients with oral or infusible DMTs were as likely to be adherent as the reference group. We found that patients with a depression diagnosis in a previous year were 5.5 percentage points less likely to be adherent to their DMTs in the current year (a marginally significant result). When patients with oral DMTs were excluded from the analysis (Table 4) because these DMTs were not available during the entire observation period, this difference in the probability of being adherent increased to 6.6 percentage points and became statistically significant. There were no statistically significant differences in current year adherence among health plans, between high and low health risk in the previous year, or having more E\&M visits in primary care. Other robustness 


\begin{tabular}{|c|c|c|c|}
\hline \multirow[t]{3}{*}{ TABLE 3} & $\begin{array}{l}\text { m Effect Prc } \\
\text { he Model }\end{array}$ & it Mo & Estimation: \\
\hline & \multicolumn{3}{|c|}{$\mathrm{MPR}>0.8$} \\
\hline & $\Delta \operatorname{Pr}(M P R>0.8)$ & $P$ Value & $95 \%$ CI \\
\hline \multicolumn{4}{|l|}{ Age category, years } \\
\hline $18-34$ & \multicolumn{3}{|c|}{ Reference } \\
\hline $35-44$ & 0.077 & 0.289 & $(-0.065-0.219)$ \\
\hline $45-54$ & 0.137 & 0.047 & $(0.002-0.272)$ \\
\hline $55-64$ & 0.161 & 0.020 & $(0.026-0.296)$ \\
\hline $65+$ & 0.186 & 0.014 & $(0.037-0.335)$ \\
\hline Female & -0.055 & 0.050 & $(-0.109-0.000)$ \\
\hline \multicolumn{4}{|l|}{ DMT type } \\
\hline Injectable, flu side effects & \multicolumn{3}{|c|}{ Reference } \\
\hline Injectable, skin side effects & -0.091 & 0.005 & $(-0.155$ to -0.028$)$ \\
\hline Orals & -0.016 & 0.769 & $(-0.120-0.089)$ \\
\hline Infusions & -0.016 & 0.706 & $(-0.098-0.067)$ \\
\hline \multicolumn{4}{|l|}{ Health plan } \\
\hline Commercial & \multicolumn{3}{|c|}{ Reference } \\
\hline Medicaid only & -0.080 & 0.206 & $(-0.205-0.044)$ \\
\hline Medicare/Medicaid & -0.290 & 0.337 & $(-0.883-0.302)$ \\
\hline Medicare only & -0.099 & 0.132 & $(-0.227-0.030)$ \\
\hline \multicolumn{4}{|l|}{ Health status, previous year } \\
\hline No/healthy/low/medium risk & \multicolumn{3}{|c|}{ Reference } \\
\hline High/very high risk & 0.002 & 0.946 & $(-0.050-0.053)$ \\
\hline $\begin{array}{l}\text { E\&M: primary care > } \\
\text { neurologist }\end{array}$ & -0.016 & 0.582 & $(-0.072-0.040)$ \\
\hline Depression, previous year & -0.056 & 0.063 & $(-0.114-0.003)$ \\
\hline Observations & 1,045 & & \\
\hline Number of patients & 698 & & \\
\hline \multicolumn{4}{|c|}{$\begin{array}{l}\triangle P r=\text { change in the probability with respect to the reference category; } C I=\text { confi- } \\
\text { dence interval; DMT=disease-modifying therapy; } E \mathcal{E} M=\text { evaluation and manage- } \\
\text { ment; } M P R=\text { medication possession ratio. }\end{array}$} \\
\hline
\end{tabular}

tests (excluding patients in Medicaid and Medicare/Medicaid managed plans or adding neighborhood socioeconomic characteristics as independent variables, both available from the corresponding author) showed no meaningful changes in the magnitude or statistical significance of these associations.

\section{Discussion}

This study finds several associations between individual characteristics of MS patients and adherence to DMTs. In general, patients aged 45 years or older were more likely to be adherent than younger patients. Women were less likely than men to be adherent; other studies have mixed evidence about the differences in adherence between men and women. ${ }^{16,29}$ Since MS is more prevalent in women, ${ }^{1}$ it is important to address patient-centered mechanisms that can be used by health care practitioners to aid in increasing adherence to DMTs in women.

Other studies have addressed the injection modality as a factor for nonadherence..$^{12,22,29}$ Our results show that the sideeffect profile of the medication also matters. Those who took a self-injectable medication whose most common side effect
TABLE 4 Random Effect Probit Model Estimation: No Oral DMTs

\begin{tabular}{|c|c|c|c|}
\hline & \multicolumn{3}{|c|}{$\mathrm{MPR}>0.8$} \\
\hline & $\Delta \operatorname{Pr}(\mathrm{MPR}>0.8)$ & $P$ Value & $95 \% \mathrm{CI}$ \\
\hline \multicolumn{4}{|l|}{ Age category, years } \\
\hline $18-34$ & \multicolumn{3}{|c|}{ Reference } \\
\hline $35-44$ & 0.100 & 0.200 & $(-0.053-0.253)$ \\
\hline $45-54$ & 0.147 & 0.049 & $(0.001-0.294)$ \\
\hline $55-64$ & 0.169 & 0.024 & $(0.022-0.316)$ \\
\hline $65+$ & 0.195 & 0.016 & $(0.037-0.353)$ \\
\hline Female & -0.051 & 0.063 & $(-0.104-0.003)$ \\
\hline \multicolumn{4}{|l|}{ DMT type } \\
\hline Injectable, flu side effects & \multicolumn{3}{|c|}{ Reference } \\
\hline Injectable, skin side effects & -0.087 & 0.006 & $(-0.150$ to -0.025$)$ \\
\hline Infusions & -0.016 & 0.693 & $(-0.094-0.063)$ \\
\hline \multicolumn{4}{|l|}{ Health plan } \\
\hline Commercial & \multicolumn{3}{|c|}{ Reference } \\
\hline Medicaid only & -0.060 & 0.324 & $(-0.179-0.059)$ \\
\hline Medicare/Medicaid & -0.297 & 0.351 & $(-0.921-0.327)$ \\
\hline Medicare only & -0.100 & 0.134 & $(-0.232-0.031)$ \\
\hline \multicolumn{4}{|l|}{ Health status, previous year } \\
\hline No/healthy/low/medium risk & \multicolumn{3}{|c|}{ Reference } \\
\hline High/very high risk & 0.011 & 0.666 & $(-0.039-0.062)$ \\
\hline $\begin{array}{l}\text { E\&M: primary care > } \\
\text { neurologist }\end{array}$ & -0.023 & 0.399 & $(-0.077-0.031)$ \\
\hline Depression, previous year & -0.067 & 0.029 & $(-0.126$ to -0.007$)$ \\
\hline Observations & 1,002 & & \\
\hline Number of patients & 667 & & \\
\hline \multicolumn{4}{|c|}{$\begin{array}{l}\Delta P r=\text { change in the probability with respect to the reference category; } C I=\text { confi- } \\
\text { dence interval; DMT = disease-modifying therapy; } E \mathcal{E} M=\text { evaluation and manage- } \\
\text { ment; } M P R=\text { medication possession ratio. }\end{array}$} \\
\hline
\end{tabular}

was injection site reactions were less adherent than those who took any other type of DMT, including other self-injectables. These differences in adherence among different types of DMTs remained during our robustness testing.

Depression is a common side effect of MS DMTs, ${ }^{1}$ is a common comorbid condition, and is a factor for nonadherence..$^{22,41}$ In the baseline model, patients with a diagnosis of depression in the previous year had marginally significant lower adherence rates in the current year than those without the diagnosis. In the models that excluded patients on oral DMTs, patients with a diagnosis of depression had statistically significant lower adherence rates.

No differences in adherence were found by having more E\&M visits in primary care than in neurology. This sample comes from an area with increased MS prevalence because of a large population of Scandinavian heritage, ${ }^{2}$ so it is likely that the primary care physicians in the sample area had a greater experience with MS guidelines and standards of care when compared with primary care physicians from other parts of the country. In the same fashion, the relatively high quality of care in the region and accessibility to care may explain why 
neighborhood or socioeconomic characteristics (i.e., being enrolled in a Medicaid or dual eligible health plan) had no association with adherence.

\section{Limitations}

This study has several limitations. First, MPRs measure patterns of medication refills, not actual medication use. ${ }^{42}$ Studies that use electronic medication monitors have less measurement error than those that use claims data to measure adherence. ${ }^{42}$ Moreover, pharmaceutical claims data on injectable medication is less reliable than for oral medication. ${ }^{43}$ However, most of the literature on adherence in MS patients uses administrative data.

Second, while this study is notable for using a broad mix of health insurance coverages, it does not use nationally representative data or multiple payer claims data. The setting of this study included a large population with Scandinavian heritage, which is linked to a higher incidence of MS. ${ }^{2}$ Therefore, patients in this study may face a health care system with more experience with MS patients than the national average. Additionally, there is a sole specialty pharmacy vendor in the health plan. That vendor monitors patients' MPRs and has performance guarantees around MPRs for MS patients on a DMT. These reasons, in addition to the "prevalent user" design, could explain the relatively high MPRs and rates of adherence. The trend toward increased use of specialty pharmacy management should increase the generalizability of our results over time.

Third, this study does not include several individual factors that the literature has shown to affect adherence such as plan design and cost-sharing information, ${ }^{23}$ self-efficacy, ${ }^{12,22}$ treatment expectations, ${ }^{44}$ lack of knowledge about MS, ${ }^{19,45}$ and cognitive deficits. ${ }^{19}$ However, the use of patient-specific random effects should adjust for these unobserved and excluded patient characteristics if these characteristics do not vary over time.

\section{Conclusions}

This study found statistically significant differences in adherence to DMTs by age, sex, type of DMT, and a comorbid diagnosis of depression. Older patients and men were more likely to be adherent. Patients with a diagnosis of depression had lower adherence to DMTs_our methods suggest that this difference is not a result of depression being a side effect of MS treatments. Compared with other self-injectable, oral, and infusible DMTs, self-injectable DMTs-the most common side effect of which is injection-site reactions-have lower adherence than any other DMTs. Improving adherence levels requires addressing sideeffect profiles of medications, shared decision making between patient and provider, and improving the comorbid mental health conditions of MS patients, which could lead to high self-efficacy and, therefore, better adherence to medication regimens.

\section{Authors}

LUCAS HIGUERA, MA, and CAROLINE S. CARLIN, PhD, Medica Research Institute, Minnetonka, Minnesota. SARAH ANDERSON, PharmD, Medica Health Plans, Minnetonka, Minnesota.

AUTHOR CORRESPONDENCE: Lucas Higuera, MA, Junior Research Fellow, Medica Research Institute, Mail Route CW105, 401 Carlson Pkwy., Minnetonka, MN 55305. Tel.: 952.992.2819; Fax: 952.992.2614; E-mail: lucas.higuera@medica.com.

\section{DISCLOSURES}

TEVA provided funding for this study and had the option to review the manuscript. The authors retained autonomy in the determination of the final content of this work.

Study concept and design were contributed by Carlin, Anderson, and Higuera. Data interpretation was primarily performed by Higuera and Carlin, along with Anderson. The manuscript was written and revised by Higuera, Carlin, and Anderson.

\section{ACKNOWLEDGMENTS}

The authors thank Beth Lindholm and Era Kim (Research Informatics, Medica Research Institute) for their help processing the claims data.

\section{REFERENCES}

1. OptumRx. Multiple sclerosis: background, new developments, key strategies. 2014. Available at: https://cdn-aem.optum.com/content/dam/optum3/ optum/en/resources/white-papers/M53018_G_MS_Insight_Report_ORx_ FINAL.pdf. Accessed September 24, 2016.

2. World Health Organization. Neurological Disorders: Public Health Challenges. Geneva: World Health Organization; 2006.

3. National Institute of Neurological Disorder and Stroke. Multiple sclerosis: hope through research. National Institute of Neurological Disorders and Stroke, National Institutes of Health. Bethesda, MD. July 2015. Available at: http://www.ninds.nih.gov/disorders/multiple_sclerosis/detail_multiple_sclerosis.htm. Accessed September 24, 2016.

4. National Multiple Sclerosis Society. Symptoms \& diagnosis. July 2015. Available at: www.nationalmssociety.org/Symptoms-Diagnosis. Accessed September 24, 2016.

5. Owens GM, Hughes BL, Lewis SJ. Optimizing new and emerging therapies for treatment and symptom management of multiple sclerosis in managed care. Am Health Drug Benefits. 2011;4(7 Suppl):S94-105.

6. Simon H. Multiple sclerosis. 2013. Available at: http://umm.edu/health/ medical/reports/articles/multiple-sclerosis. Accessed September 24, 2016.

7. Goodin DS, Frohman EM, Garmany GP Jr, et al. Disease modifying therapies in multiple sclerosis: report of the Therapeutics and Technology Assessment Subcommittee of the American Academy of Neurology and the MS Council for Clinical Practice Guidelines. Neurology. 2002;58(2):169-78.

8. Reuben DB, Tinetti ME. Goal-oriented patient care-an alternative health outcomes paradigm. N Engl J Med. 2012;366(9):777-79.

9. Barry MJ, Edgman-Levitan S. Shared decision making — pinnacle of patient-centered care. N Engl J Med. 2012;366(9):780-81.

10. Happe LE. Choosing the best treatment for multiple sclerosis: comparative effectiveness, safety, and other factors involved in disease-modifying therapy choice. Am J Manag Care. 2013;19(17 Suppl):S332-42. 
11. Frazee S, Miller S, Nease R, Stettin G. 2011 Drug trend report. ExpressScripts. April 2012. Available at: http://www.express-scripts.com/ research/research/dtr/archive/2011/dtrFinal.pdf. Accessed September 24, 2016.

12. Mohr D, Boudewyn A, Likosky W, Levine E, Goodkin D. Injectable medication for the treatment of multiple sclerosis: the influence of selfefficacy expectations and infection anxiety on adherence and ability to selfinject. Ann Behav Med. 2001;23(2):125-32.

13. Halpern R, Agarwal S, Borton L, Oneacre K, Lopez-Bresnahan M. Adherence and persistence among multiple sclerosis patients after one immunomodulatory therapy failure: retrospective claims analysis. Adv Ther. 2011;28(9):761-75.

14. Sabaté E. ed. Adherence to Long-Term Therapies: Evidence for Action. Geneva: World Health Organization; 2003. Available at: http://www.who. $\mathrm{int} / \mathrm{chp} /$ knowledge/publications/adherence_report/en/index.html. Accessed September 24, 2016

15. Tremlett H, van der Mei I, Pittas F, et al. Adherence to the immunomodulatory drugs for multiple sclerosis: contrasting factors affect stopping drug and missing doses. Pharmacoepidemiol Drug Saf. 2008;17(6):565-76.

16. Devonshire V, Lapierre Y, Macdonell R, et al. The Global Adherence Project (GAP): a multicenter observational study on adherence to diseasemodifying therapies in patients with relapsing-remitting multiple sclerosis. Eur J Neurol. 2011;18(1):69-77.

17. Patti F. Optimizing the benefit of multiple sclerosis therapy: the importance of treatment adherence. Patient Prefer Adherence. 2010;4:1-9.

18. Bruce JM, Hancock LM, Arnett P, Lynch S. Treatment adherence in multiple sclerosis: association with emotional status, personality, and cognition. J Behav Med. 2010;33(3):219-27.

19. Holland N, Wiesel P, Cavallo P, et al. Adherence to disease-modifying therapy in multiple sclerosis: part I. Rehabil Nurs. 2001;26(5):172-76.

20. Saunders C, Caon C, Smrtka J, Shoemaker J. Factors that influence adherence and strategies to maintain adherence to injected therapies for patients with multiple sclerosis. J Neurosci Nurs. 2010;42(5 Suppl):S10-18.

21. Koudriavtseva T, Onesti E, Pestalozza I, Sperduti I, Jandolo B. The importance of physician-patient relationship for improvement of adherence to long-term therapy: data of survey in a cohort of multiple sclerosis patients with mild and moderate disability. Neurol Sci. 2012;33(3):575-84

22. Caon C, Saunders C, Smrtka J, Baxter N, Shoemaker J. Injectable disease-modifying therapy for relapsing-remitting multiple sclerosis: a review of adherence data. J Neurosci Nurs. 2010;42(5 Suppl):S5-9.

23. Dor A, Lage MJ, Tarrants ML, Castelli-Haley J. Cost sharing, benefit design, and adherence: the case of multiple sclerosis. Adv Health Econ Health Serv Res. 2010;22:175-93.

24. Lafata JE, Cerghet M, Dobie E, et al. Measuring adherence and persistence to disease-modifying agents among patients with relapsing remitting multiple sclerosis. J Am Pharm Assoc (2003). 2008;48(6):752-57.

25. Treadaway K, Cutter G, Salter A, et al. Factors that influence adherence with disease-modifying therapy in MS. J Neurol. 2009;256(4):568-76.

26. Meyniel C, Spelman T, Jokubaitis VG, et al. Country, sex, EDSS change and therapy choice independently predict treatment discontinuation in multiple sclerosis and clinically isolated syndrome. PLoS One. 2012;7(6):e38661.

27. Río J, Porcel J, Téllez N, et al. Factors related with treatment adherence to interferon beta and glatiramer acetate therapy in multiple sclerosis. Mult Scler. 2005;11(3):306-09.
28. Lad SP, Chapman CH, Vaninetti M, Steinman L, Green A, Boakye M. Socioeconomic trends in hospitalization for multiple sclerosis. Neuroepidemiology. 2010;35(2):93-99.

29. Halpern R, Agarwal S, Dembek C, Borton L, Lopez-Bresnahan M. Comparison of adherence and persistence among multiple sclerosis patients treated with disease-modifying therapies: a retrospective administrative claims analysis. Patient Prefer Adherence. 2011;5:73-84

30. Agashivala N, Wu N, Abouzaid S, et al. Compliance to fingolimod and other disease modifying treatments in multiple sclerosis patients, a retrospective cohort study. BMC Neurol. 2013;13:138.

31. Bergvall N, Petrilla AA, Karkare SU, et al. Persistence with and adherence to fingolimod compared with other disease-modifying therapies for the treatment of multiple sclerosis: a retrospective U.S. claims database analysis. J Med Econ. 2014;17(10):696-707.

32. Kleinman NL, Beren IA, Rajagopalan K, Brook RA. Medication adherence with disease modifying treatments for multiple sclerosis among U.S employees. J Med Econ. 2010;13(4):633-40.

33. Kardas P, Lewek P, Matyjaszczyk M. Determinants of patient adherence: a review of systematic reviews. Front Pharmacol. 2013;4:91

34. Cohen BA, Coyle PK, Leist T, Oleen-Burkey MA, Schwartz M, Zwibel H. Therapy optimization in multiple sclerosis: a cohort study of therapy adherence and risk of relapse. Mult Scler Relat Disord. 2015;4(1):75-82.

35. Maciejewski ML, Bryson CL, Wang V, Perkins M, Liu CF. Potential bias in medication adherence studies of prevalent users. Health Serv Res. 2013;48(4):1468-86.

36. Sikka R, Xia F, Aubert RE. Estimating medication persistency using administrative claims data. Am J Manag Care. 2005;11(7):449-57.

37. Gibbons RD, Hedeker D. Application of random-effects probit regression models. J Consult Clin Psychol. 1994;62(2):285-96.

38. Davies HTO, Crombie IK, Tavakoli M. When can odds ratios mislead? BMJ. 1998;316(7136):989-91

39. U.S. Food and Drug Administration. FDA approves new multiple sclerosis treatment Aubagio. September 12, 2012. Available at: http://www.fda.gov/ NewsEvents/Newsroom/PressAnnouncements/ucm319277.htm. Accessed September 24, 2016.

40. U.S. Food and Drug Administration. FDA approves new multiple sclerosis treatment: Tecfidera. March 27, 2013. Available at: http://www.fda.gov/ NewsEvents/Newsroom/PressAnnouncements/ucm345528.htm. Accessed September 24, 2015.

41. Costello K, Kennedy P, Scanzillo J. Recognizing nonadherence in patients with multiple sclerosis and maintaining treatment adherence in the long term. Medscape J Med. 2008;10(9):225.

42. Osterberg L, Blaschke T. Adherence to medication. N Engl J Med. 2005;353(5):487-97.

43. Fairman KA, Motheral B. Evaluating medication adherence: which measure is right for your program? J Manag Care Pharm. 2000;6(6):499-506. Available at: http://www.jmcp.org/doi/abs/10.18553/jmcp.2000.6.6.499.

44. Klauer T, Zettl U. Compliance, adherence, and the treatment of multiple sclerosis. J Neurol. 2008;255(6):87-92.

45. Holland N, Wiesel P, Cavallo P, et al. Adherence to disease-modifying therapy in multiple sclerosis: part II. Rehabil Nurs. 2001;26(6):221-26. 